\title{
Augmented input estimation in multiple maneuvering target tracking
}

\author{
HADAEGH Mahmoudreza ${ }^{1}, \mathrm{KHALOOZADEH} \mathrm{Hamid}^{2, *}$, and BEHESHTI Mohammadtaghi ${ }^{3}$ \\ 1. Department of Electrical and Computer Engineering, Science and Research Branch, Islamic Azad University, \\ Tehran 1477893855, Iran; 2. Department of Systems and Control, K. N. Toosi University of Technology, Tehran 1969764499, Iran; \\ 3. Department of Electrical Engineering, Control Group, Tarbiat Modares University, Tehran 14115111, Iran
}

\begin{abstract}
This paper presents augmented input estimation (AIE) for multiple maneuvering target tracking. Multi-target tracking (MTT) is based on two main parts, data association and estimation. In data association (DA), the best observations are assigned to the considered tracks. In real conditions, the number of observations is more than targets and also locations of observations are often so scattered that the association between targets and observations cannot be done simply. In this case, for general MTT problems with unknown numbers of targets, we present a Markov chain MonteCarlo DA (MCMCDA) algorithm that approximates the optimal Bayesian filter with low complexity in computations. After DA, estimation and tracking should be done. Since in general cases, many targets can have maneuvering motions, then AIE is proposed to cover both the non-maneuvering and maneuvering parts of motion and the maneuver detection procedure is eliminated. This model with an input estimation (IE) approach is a special augmentation in the state space model which considers both the state vector and the unknown input vector as a new augmented state vector. Some comparisons based on the Monte-Carlo simulations are also made to evaluate the performances of the proposed method and other older methods in MTT.
\end{abstract}

Keywords: multi-target tracking (MTT), Markov chain Monte-Carlo data association (MCMCDA), data association (DA), augmented input estimation (AIE).

DOI: $10.21629 / J S E E .2019 .05 .03$

\section{Introduction}

Multiple maneuvering target tracking is the determination of the present and often future states (position, velocity and acceleration) of some moving targets from noisy measurements of their present states. Tracking maneuvering targets is an important problem complicated by the fact that the radar cannot directly measure target velocity and acceleration [1]. As we mentioned in abstract, multi-target tracking (MTT) is based on two main parts, data association (DA)

Manuscript received December 17, 2018.

*Corresponding author. and estimation, and we will describe it in details why DA is needed in MTT problems. But at first we want to review different ideas in estimation and tracking.

Many different tracking filters to track a maneuvering target have been considered in the literature. Two different approaches that have been widely used to handle the case of unknown target maneuvers are interacting multiple models (IMM) filtering [2] and input estimation (IE).

The IMM filter consists of a first-degree model for the target non-maneuvering motion and one or two seconddegree models with different process noise levels for the maneuvering motions. It utilizes multiple different models to estimate the state of single target and combines the estimates of all the models with non-zero model probability. This model has good performance when the modes accurately represent the true accelerations and is relatively robust to small modeling errors, but may results in some performance degradation because of over-modeling in the non-maneuvering case and also its computational complexity increases linearly with the number of maneuver modes.

The IE approach for tracking a maneuvering target was proposed by Chan et al. [3]. What we do in this approach for maneuver detection is to use least squares estimation for identification of acceleration magnitude. Then, for compensating the target state estimation, the estimated acceleration will be inserted in the structure of the standard Kalman filter. When no maneuver occurs, the Kalman filter is used alone. Although this algorithm is an effective tool in some situations but because of constant input assumption, it confronts significant problems. Totally, what happens in this algorithm is estimating the unknown control input $\boldsymbol{u}(t)$ and then using the estimated input $\widehat{\boldsymbol{u}}(t)$ for state estimating. Many improvements on the original IE algorithm were introduced in [4-6] by relaxing certain assumptions. In 
this paper a new IE model will be presented for problems in maneuvering targets tracking. In this model, acceleration is added as a new term to the state equation. This model is an augmented state space model considering both previous elements of standard state vectors and unknown input vectors. This proposed algorithm is suitable in both cases of maneuver and non-maneuver modes.

Now we want to answer the question in the first paragraph of introduction: why DA is needed in MTT problems? In MTT, the number of measurements and observations is often more than targets and their locations are so scattered that we cannot determine which measurement is originated from which target certainly. Then we cannot divide the MTT problem into some parallel single target tracking and before tracking process, we have to use a procedure to determine the best assignments between measurements and targets. In other words, the duty of the DA problem is to determine which measurements and targets are related to each other. Therefore, partition of measurements originated from a single target or clutter shall be separated in different groups [7]. Also it should be noted that uncertainty is unavoidable in correct associations.

The multiple hypothesis tracking (MHT) algorithm [8] and the joint probability DA (JPDA) algorithm [9] are the most famous maximum a posteriori (MAP) and Bayesian estimators respectively. MHT is a kind of MTT algorithm that maintains the history of associations between previous measurements and related targets. With a new set of measurements, new sets of associations are formed based on previous associations. Finally, the algorithm returns highest posterior associations as a solution. But in JPDA as the suboptimal Bayesian filter, all possible associations are enumerated and the association probabilities $\beta_{j k}$ are computed too. $\beta$ is the probability value for association between the $j$ th measurement and the $k$ th track.

But in this paper for the DA problem, Markov chain Monte-Carlo DA (MCMCDA) is used for tracking multi targets in real mode. MCMCDA unlike MHT and JPDA converges to optimal Bayesian solution with unbounded data resources. It is explicit that in MCMCDA, the Markov chain Monte-Carlo (MCMC) sampling method is used instead of considering all possible associations. MCMC can be used to approximate $\beta$ coefficients in JPDA and also shows better performance in comparison with Fitzgerald's cheap JPDA [10-14]. MCMCDA can be applied to the different cases of DA problems because of its ability to merging false alarms, missing measurements and tracking targets with unknown times of entrance and exit.

JPDA has proved very effective in cluttered environments but it can only work in the situation of fixed number of targets. Also, MHT can work in both situations of fixed and unknown number of targets but its computational complexity increases when the number of hypothesis increases. While MCMCDA can do DA in two cases of fixed and unknown number of targets and in [7] it was shown that the performance of MCMCDA compared with JPDA and MHT was better and also was more rapid than these two older methods.

The reminder of this paper is structured as follows. Principles of MTT and description of MCMCDA are summarized in Section 2. In Section 3, augmented IE (AIE) for multiple maneuvering target tracking as the proposed model and related computations are presented and finally Section 4 includes the application of proposed model and discussion of results and comparison to other models.

\section{MTT}

\subsection{Principles and formulations}

Let $T_{\text {fin }} \in \mathbb{Z}^{+}$be the duration of surveillance and $T$ is the sampling time. Let $J$ be the number of targets that appear in the surveillance region $R$ during the surveillance period. Each target $j$ moves in $R$ for some unknown duration $\left[t_{i}^{j}, t_{f}^{j}\right] \subset\left[T_{\text {init }}, T_{\text {fin }}\right]$. Every time $t$ is a multiple of sampling time $T\left(t=k T, t_{i}^{j}=k_{i} T\right.$ and $\left.t_{f}^{j}=k_{f} T\right)$. Some important parameters are used in the MTT problem that are $p_{z}, \lambda_{b} V, p_{d}$ and $\lambda_{f} V . p_{z}$ is the disappearance probability of targets so it persists with the probability $1-p_{z}$. Each time the number of targets arising in the volume $V$ has a Poisson distribution with a parameter $\lambda_{b} V$ where $\lambda_{b}$ is the birth rate of new targets per unit time and unit volume. $V$ is the volume of $R$. Also in the presence of a target, $p_{d}$ is the detection probability of a noisy observation of the target state. It means that with $1-p_{d}$, target detection is not possible and called missing observation. Distribution of false alarms is based on Poisson distribution with the parameter $\lambda_{f} V . \lambda_{f}$ is the false alarm rate per unit time and unit volume.

Let $\boldsymbol{A}^{j}: \mathbb{R}^{n x} \rightarrow \mathbb{R}^{n x}$ be the state equation transient matrix in the discrete-time mode for the $j$ th target. The state variable dimension is $n_{x}$ and the $j$ th target state is shown with $\boldsymbol{X}^{j}(k T) \in \mathbb{R}^{n x}$ at the time $k T$ for $k=$ $k_{i}^{j}, \ldots, k_{f}^{j}-1$. The movement equation for this target is according to

$$
\boldsymbol{X}^{j}((k+1) T)=\boldsymbol{A}^{j}\left(\boldsymbol{X}^{j}(k T)\right)+\boldsymbol{C}^{j} \boldsymbol{u}^{j}(k T)+\boldsymbol{B}^{j} \boldsymbol{\omega}^{j}(k T)
$$

that since now, in equations we use $k$ instead of $k T$. The acceleration input vectors are $\boldsymbol{u}^{j}(k)=\left[\begin{array}{ll}a_{x}^{j} & a_{y}^{j}\end{array}\right]^{\mathrm{T}}$. It is assumed that the acceleration $\boldsymbol{u}^{j}$ is a commonly unknown input which models the target maneuvers, when there is no maneuver, $\boldsymbol{u}$ is zero. Also $\boldsymbol{\omega}^{j}(k) \in \mathbb{R}^{n x}$ is white noises in process. Let $n(k)$ be the number of all noisy observations 
and false alarms at the time $k T$. Let $\boldsymbol{y}^{m}(k) \in \mathbb{R}^{n y}$ be the $m$ th observation at time $k$ for $m=1, \ldots, n(k)$ and $n_{y}$ is the observation vector dimension. If the target is detected, it can only produce one observation at each sampling time. Also $\boldsymbol{H}^{m}: \mathbb{R}^{n x} \rightarrow \mathbb{R}^{n y}$ is the observation model and the observations are produced as follows:

$$
\boldsymbol{y}^{m}(k)=\left\{\begin{array}{c}
\boldsymbol{H}^{m}\left(\boldsymbol{x}^{j}(k)\right)+\boldsymbol{v}^{m}(k), \\
\boldsymbol{y}^{m}(k) \text { is from } \boldsymbol{x}^{j}(k) \\
\boldsymbol{u}_{f}(k), \text { otherwise }
\end{array}\right.
$$

where $\boldsymbol{v}^{m}(k) \in \mathbb{R}^{n y}$ is measurement white noises and $\boldsymbol{u}_{f}(k) \sim \operatorname{Unif}(R)$ is a random process for false alarms. The goal of MTT is to estimate $J,\left\{t_{i}^{j}, t_{f}^{j}\right\}$ and $\left\{\boldsymbol{x}^{j}(t)\right.$ : $\left.t_{i}^{j} \leqslant t \leqslant t_{f}^{j}\right\}(j=1, \ldots, J)$ from noisy observations.

Let $\boldsymbol{Y}(k)=\left\{\boldsymbol{y}^{m}(k): m=1, \ldots, n(k)\right\}$ be all measurements at time $k$ and $\boldsymbol{Y}=\left\{\boldsymbol{Y}(k): T_{\text {init }} \leqslant k T \leqslant T_{\text {fin }}\right\}$ be all measurements from $t=T_{\text {init }}$ to $t=T_{\text {fin }}$. Let $\Omega$ be a collection of partitions of $\boldsymbol{Y}$ such that, for $\boldsymbol{\omega} \in \boldsymbol{\Omega}$, $\boldsymbol{\omega}=\left\{\boldsymbol{\tau}_{0}, \boldsymbol{\tau}_{1}, \ldots, \boldsymbol{\tau}_{J}\right\}$, where $\boldsymbol{\tau}_{0}$ and $\boldsymbol{\tau}_{j}(j=1, \ldots, J)$ are set of false alarms and set of measurements respectively. $\boldsymbol{\omega}$ as an association event is defined as follows [7]:

$$
\boldsymbol{\omega}=\left\{\boldsymbol{\tau}_{0}, \boldsymbol{\tau}_{1}, \ldots, \boldsymbol{\tau}_{J}\right\}
$$

where

$$
\left\{\begin{array}{l}
\bigcup_{j=0}^{J} \boldsymbol{\tau}_{j}=\boldsymbol{Y}, \boldsymbol{\tau}_{i} \cap \boldsymbol{\tau}_{j}=\varnothing, \quad i \neq j \\
\boldsymbol{\tau}_{0} \text { is a set of false alarms } \\
\left|\boldsymbol{\tau}_{j} \cap \boldsymbol{Y}(t)\right| \leqslant 1, \quad j=1, \ldots, J ; t=T_{\text {init }}, \ldots, T_{\text {fin }} \\
\left|\boldsymbol{\tau}_{j}\right| \geqslant 2, \quad j=1, \ldots, J
\end{array} .\right.
$$

Fig. 1 shows an association example where $J$ is the number of tracks. $\boldsymbol{\tau}_{j}$ is a track in the condition of no confusion and really the set of estimated states derived from observations $\boldsymbol{\tau}_{j}$ makes the actual track. We assume there are at least two observations for a track since it cannot be distinguished with a single observation from a false alarm. Now we can define $n_{e}(k-1)$ as the number of targets at the time $(k-1) T$ and $n_{z}(k)$ as the number of terminated targets at the time $k T$.

New targets at time $k$ is shown with $n_{b}(k)$ and $n_{c}(k)=$ $n_{e}(k-1)-n_{z}(k)$ shows the number of targets from the time $(k-1) T$ without termination at the time $k T . n_{d}(k)$ is used as the number of detected actual targets at time $k$ and $n_{u}(k)=n_{c}(k)+n_{b}(k)-n_{d}(k)$ for undetected targets. Finally, $n_{f}(k)=n(k)-n_{d}(k)$ shows the number of false alarms. According to Bayes rule, the posterior $\omega$ is as follows [9]:

$$
\begin{gathered}
P(\boldsymbol{\omega} \mid \boldsymbol{Y}) \propto P(\boldsymbol{\omega}) P(\boldsymbol{Y} \mid \boldsymbol{\omega}) \propto \\
\prod_{k=1}^{k_{f}} p_{z}^{n_{z}(k)}\left(1-p_{z}\right)^{n_{c}(k)} p_{d}^{n_{d}(k)}\left(1-p_{d}\right)^{n_{u}(k)}
\end{gathered}
$$

$$
\left(\lambda_{b} V\right)^{n_{b}(k)}\left(\lambda_{f} V\right)^{n_{f}(k)} P(\boldsymbol{Y} \mid \boldsymbol{\omega})
$$

where $P(\boldsymbol{Y} \mid \boldsymbol{\omega})$ is the likelihood of observations $\boldsymbol{Y}$ given $\boldsymbol{\omega}$, computed according to the state space and measurement equations. In [7], an example for computation of $P(\boldsymbol{Y} \mid \boldsymbol{\omega})$ can be seen for the linear state space and the measurement model. In most multi-target algorithms, the parameters $p_{z}$, $p_{d}, \lambda_{b}$ and $\lambda_{f}$ are so effective and used abundantly $[9,15]$.

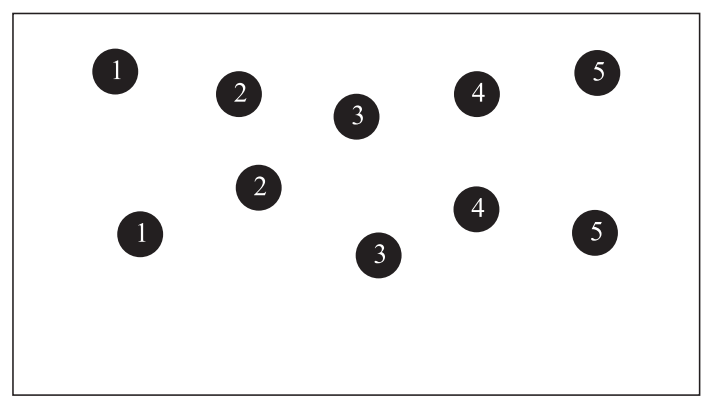

(a) Observations and false alarms

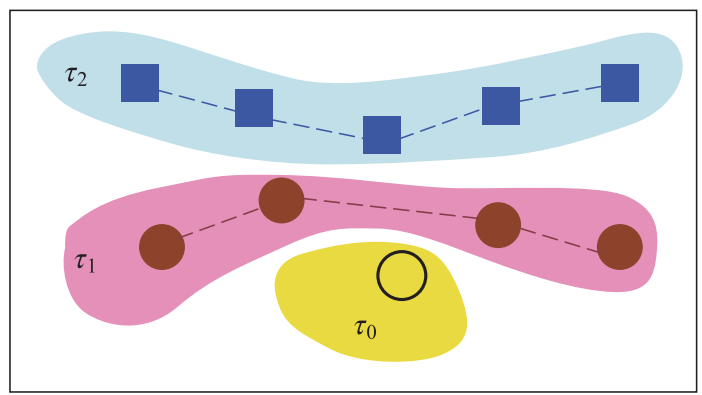

(b) A sample of association $\omega$

Fig. 1 A set of observations and false alarms at consecutive times and their association method

Bayesian and MAP models are two famous approaches used in MTT problems [14]. But because of convenience in the MAP approach, we prefer to take this approach in this paper.

What happens in the MAP approach is maximizing $P(\boldsymbol{\omega} \mid \boldsymbol{Y})$ with a suitable partition of observations and estimating the targets states based on this partition. In the MAP approach, we try to seek $\widehat{\omega}$ according to

$$
\widehat{\boldsymbol{\omega}}=\arg \max _{\boldsymbol{\omega} \in \Omega} P(\boldsymbol{\omega} \mid \boldsymbol{Y}) .
$$

\subsection{MCMC DA algorithm}

In this section, the MCMC sampler for the MTT problem will be explained. In this method a Markov chain $M$ is utilized to producing samples from a distribution $\pi$. But the states of $M$ are $\boldsymbol{\omega}$ with stationary distribution $\pi(\boldsymbol{\omega})$. If the start point is on $\boldsymbol{\omega} \in \boldsymbol{\Omega}, \boldsymbol{\omega}^{\prime} \in \boldsymbol{\Omega}$ will be proposed according to $q\left(\boldsymbol{\omega}, \boldsymbol{\omega}^{\prime}\right)$ and the acceptance probability $A\left(\boldsymbol{\omega}, \boldsymbol{\omega}^{\prime}\right)$ determines if the move is accepted or not.

$$
A\left(\boldsymbol{\omega}, \boldsymbol{\omega}^{\prime}\right)=\min \left(1, \frac{\pi\left(\boldsymbol{\omega}^{\prime}\right) q\left(\boldsymbol{\omega}^{\prime}, \boldsymbol{\omega}\right)}{\pi(\boldsymbol{\omega}) q\left(\boldsymbol{\omega}, \boldsymbol{\omega}^{\prime}\right)}\right)
$$


otherwise the sampler stays at $\boldsymbol{\omega}$. With this construction, the detailed balance condition is satisfied, i.e., for all $\omega$, $\omega^{\prime} \in \Omega$,

$$
Q\left(\boldsymbol{\omega}, \boldsymbol{\omega}^{\prime}\right)=\pi(\boldsymbol{\omega}) P\left(\boldsymbol{\omega}, \boldsymbol{\omega}^{\prime}\right)=\pi\left(\boldsymbol{\omega}^{\prime}\right) P\left(\boldsymbol{\omega}^{\prime}, \boldsymbol{\omega}\right) .
$$

The transition probability from $\boldsymbol{\omega}$ to $\boldsymbol{\omega}^{\prime}$ is $P\left(\boldsymbol{\omega}, \boldsymbol{\omega}^{\prime}\right)=$ $q\left(\boldsymbol{\omega}, \boldsymbol{\omega}^{\prime}\right) A\left(\boldsymbol{\omega}, \boldsymbol{\omega}^{\prime}\right)$. Therefore, $M$ is a reversible Markov chain and according to ergodic theorem, $M$ converges to the stationary distribution if it is irreducible and aperiodic [16].

The algorithm of MCMCDA is described in Table 1. The MCMC algorithm with the state space $\boldsymbol{\Omega}$ described in Section 2.1 and also the stationary distribution in posterior (3) are the main core of the MCMCDA algorithm. The algorithm is described as follows.

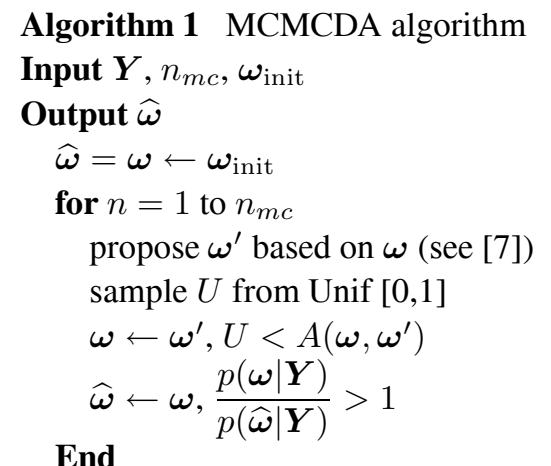

Five types of move pairs are proposed for the MCMCDA distribution that are birth/death move, split/merge move, extension/reduction move, track update move and finally track switch move. Details for each move are described in [15] and these moves are illustrated in Fig. 2. Dotted lines and hollow circles show the associations and false alarms respectively. In each move, a new association event is proposed as a modification of the current association [17].
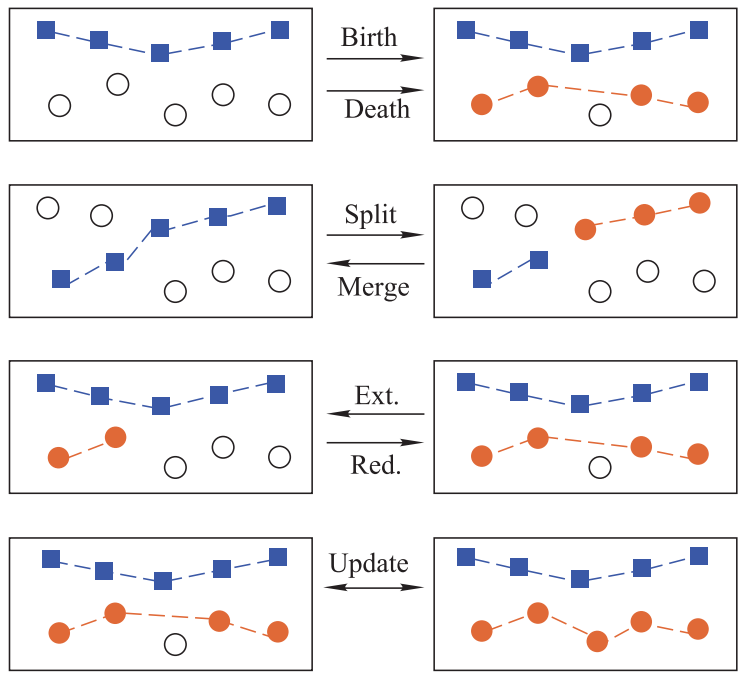

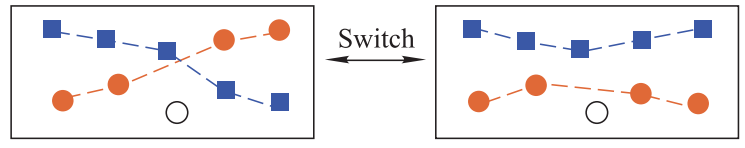

Fig. 2 MCMCDA moves

The set of all observations $\boldsymbol{Y}$, the number of samples $n_{m c}$, and the initial state $\boldsymbol{\omega}_{\text {init }}$ are the inputs for the MCMCDA algorithm. The definition of the acceptance probability $A\left(\boldsymbol{\omega}, \boldsymbol{\omega}^{\prime}\right)$ is shown in (5) with $\pi(\boldsymbol{\omega})=P(\boldsymbol{\omega} \mid \boldsymbol{Y})$ from (3). The MCMC algorithm is used to derive an optimal solution for associating problem. In addition, in this algorithm instead of all associations $\{\boldsymbol{\omega}(n)\}_{n=1}^{n_{m c}}$, only the maximum posterior partition $\widehat{\omega}$ is used simply. It should be noted that the new $\boldsymbol{\omega}$ in MCMC is constructed according to the proposal distribution $q\left(\boldsymbol{\omega}, \boldsymbol{\omega}^{\prime}\right)$ and storing previously visited states is not needed.

Because of Metropolis-Hastings kernel (5), the detailed balance condition is satisfied in the transition models of the MCMCDA algorithm. Also, since the mentioned Markov chain is irreducible and aperiodic [14], according to the ergodic theorem, this chain converges to its stationary distribution [18-20].

\section{AIE in MTT}

AIE in MTT is the new idea and main purpose of this paper. The first part of this algorithm is DA based on MCMCDA and the second part is AIE as estimation part of the problem.

\subsection{Two simplifier assumptions in DA}

In DA, we can make two simplifier and reasonable assumptions:

Assumption 1 The maximum distance between two consecutive observations associated to a specific track is less than some $D$.

Assumption 2 The number of consecutive missing observations of any track is less than some $\bar{d}$.

In Assumption 1, for two cases of maneuver and nonmaneuver motions, two different values of maximum distance should be considered. In non-maneuver, for any target, since the maximum directional velocity in $R$ is less than some $\overline{\boldsymbol{v}}_{d}$, the $\boldsymbol{D}_{v-\max }=\overline{\boldsymbol{v}}_{d} T$ will be the maximum distance. But in maneuvering case, for any target, the maximum directional acceleration in $R$ should be less than some $\overline{\boldsymbol{a}}_{d}$ and therefore derivation of $\boldsymbol{D}_{a-\max }$ according to $\overline{\boldsymbol{a}}_{d}$ and also initial velocity $\overline{\boldsymbol{v}}_{d}$ are shown as follows.

Algorithm 2 Calculation of $\boldsymbol{D}_{a-\max }$.

Attention:

(i) $\boldsymbol{D}_{a-\max }=$ maximum accepted distance between two consecutive associated observations. 
(ii) $\mathrm{sqrt}=\sqrt{ }$

$$
\begin{gathered}
\overline{\boldsymbol{a}}_{d}=\left[\begin{array}{ll}
a_{x} & a_{y}
\end{array}\right]^{\mathrm{T}}, \quad \overline{\boldsymbol{v}}_{d}=\left[\begin{array}{ll}
v_{0 x} & v_{0 y}
\end{array}\right]^{\mathrm{T}} \\
\boldsymbol{d}_{t}=\left[\begin{array}{ll}
X_{t} & Y_{t}
\end{array}\right]^{\mathrm{T}}, \quad \boldsymbol{d}_{0}=\left[\begin{array}{ll}
X_{0} & Y_{0}
\end{array}\right]^{\mathrm{T}} \\
\boldsymbol{d}_{t}=\frac{1}{2} \boldsymbol{a}_{T} t^{2}+\boldsymbol{v}_{T} t+\boldsymbol{d}_{0} \\
X_{k}=\frac{1}{2} a_{x}(k T)^{2}+v_{0 x}(k T)+X_{0} \\
Y_{k}=\frac{1}{2} a_{y}(k T)^{2}+v_{0 y}(k T)+Y_{0} \\
X_{k+1}-X_{k}=\frac{1}{2} a_{x}(2 k+1) T^{2}+v_{0 x} T \\
Y_{k+1}-Y_{k}=\frac{1}{2} a_{y}(2 k+1) T^{2}+v_{0 y} T
\end{gathered}
$$

Therefore, $\boldsymbol{D}_{a-\max }$ can be derived as follows.

$$
\begin{gathered}
\operatorname{norm}\left|P_{k+1}-P_{k}\right|=\boldsymbol{D}_{a-\max }= \\
\operatorname{sqrt}\left[\left(\frac{1}{2}(2 k+1) \operatorname{norm}\left(\bar{a}_{d}\right) T^{2}\right)^{2}+\right. \\
\left.\left(\operatorname{norm}\left(\overline{\boldsymbol{v}}_{d}\right) T\right)^{2}+(2 k+1) T^{3} \bar{a}_{d}^{\mathrm{T}} \cdot \overline{\boldsymbol{v}}_{d}\right]
\end{gathered}
$$

According to Assumption 2, the probability of object observation at least once out of $s$ consecutive measurement is $p_{d t}(s)=1-\left(1-p_{d}\right)^{s}$. For example, in the case of $p_{d}=0.7$ and $p_{d t}=0.99$, a track with a probability more than 0.99 can be detected (for $\bar{d} \geqslant 4$ ). These two simplifier assumptions and also definition of $\Omega$ are used for computational efficiency.

According to the above two assumptions, generation of two observations at different times from the same target can be determined. For example, in Fig. 3, $\boldsymbol{y}_{T}^{1}$ is a measurement made at the time $t=T$. Then other measurement for this target at the time $t=2 T$ should be in the range of $d=d_{T}$ centered at $\boldsymbol{y}_{T}^{1}$. Accordingly, $\boldsymbol{y}_{2 T}^{1}$ can be accepted as a measurement for this target but $\boldsymbol{y}_{2 T}^{2}$ cannot. As can be seen from Fig. 3, the measurements are represented with circles (positions of targets in 2D).

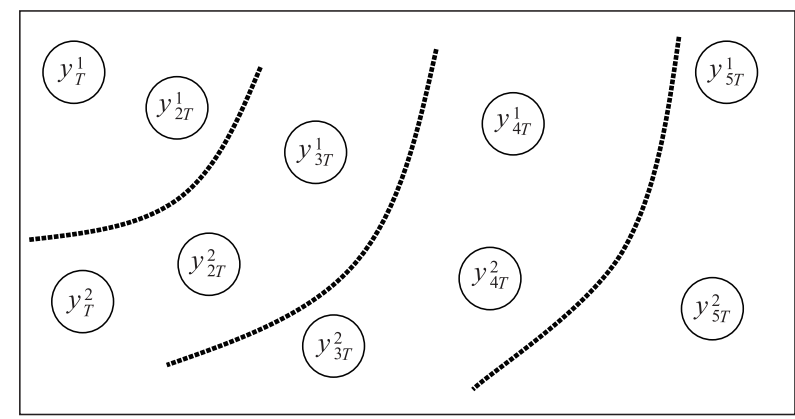

Fig. 3 An example to illustrate Assumptions 1 and 2
Now we present a relation for this concept based on the following data structure. $L\left(\boldsymbol{y}_{k T}, k T, T\right)$ can be introduced as a set of all measurements at the time $(k+1) T$ that can be associated with a measurement $\boldsymbol{y} \in \mathbb{R}^{n y}$ at the time $k T$, i.e.,

$$
\begin{gathered}
L\left(\boldsymbol{y}_{k T}, k T, T\right)= \\
\left\{\boldsymbol{y}_{(k+1) T}^{j} \in \boldsymbol{y}_{(k+1) T}: \varphi\left(\boldsymbol{y}_{k T}, \boldsymbol{y}_{(k+1) T}^{j}\right) \leqslant \boldsymbol{D}_{\max }\right\} .
\end{gathered}
$$

$\varphi: \mathbb{R}^{n y} \times \mathbb{R}^{n y} \rightarrow \mathbb{R}$ is an appropriate metric, e.g., for a Cartesian coordinate system, and $\varphi$ is induced by the Euclidean norm. $\boldsymbol{D}_{\max }$ can be in one of the forms of $\boldsymbol{D}_{v-\max }$ or $\boldsymbol{D}_{a-\max }$. Equation (8) is described in more details according to the algorithm described below. In Fig. 3, $L\left(\boldsymbol{y}_{T}^{1}, t=T, d=d_{T}\right)=\left\{\boldsymbol{y}_{2 T}^{1}\right\}, L\left(\boldsymbol{y}_{T}^{1}, t=T, d=\right.$ $\left.d_{2 T}\right)=\left\{\boldsymbol{y}_{3 T}^{1}\right\}$ and $L\left(\boldsymbol{y}_{T}^{1}, t=T, d=d_{3 T}\right)=\left\{\boldsymbol{y}_{4 T}^{1}, \boldsymbol{y}_{4 T}^{2}\right\}$. $L\left(\boldsymbol{y}_{k T}, k T, T\right)$ is so effective to make the algorithm more scalable and because of easier computations in proposal distribution, distant observations are considered separately.

Algorithm 3 Association for two consecutive observations.

$$
\begin{aligned}
& \text { for all } \boldsymbol{y}_{(k+1) T}^{m} \in \boldsymbol{y}_{(k+1) T} \\
& \quad \text { if } \varphi\left(\boldsymbol{y}_{k T}, \boldsymbol{y}_{(k+1) T}^{m}\right) \leqslant \boldsymbol{D}_{v-\max }
\end{aligned}
$$

It means that non-maneuver motion has priority to maneuver motion and therefore $\boldsymbol{y}_{(k+1) T}^{m}$ can be associated to $\boldsymbol{y}_{k T}$.

else if $\varphi\left(\boldsymbol{y}_{k T}, \boldsymbol{y}_{(k+1) T}^{m}\right) \leqslant \boldsymbol{D}_{a-\max }$

It means that non-maneuver approach does not work and target motion may be in maneuver case. Then $\boldsymbol{y}_{(k+1) T}^{m}$ can be associated to $\boldsymbol{y}_{k T}$.

\section{Else}

No observe in $(k+1) T$ can be associated to $\boldsymbol{y}_{k T}$.

\section{End}

\section{End}

Gating technique in MHT is the same as what we do in MCMCDA. But in MCMCDA, $L\left(\boldsymbol{y}_{k T}, k T, T\right)$ for a given set of observations is fixed. Also for consistent computation of proposal distribution $q\left(\boldsymbol{\omega}, \boldsymbol{\omega}^{\prime}\right), L\left(\boldsymbol{y}_{k T}, k T, T\right)$ is considered fixed. Fig. 3 shows an example about simplifier assumptions.

\subsection{AIE}

The simplest standard state space equation of a target motion is in the form of

$$
\boldsymbol{X}(k+1)=\boldsymbol{A}(k) \boldsymbol{X}(k)+\boldsymbol{B}(k) \boldsymbol{\omega}(k)
$$

that is a non-maneuver dynamic case and $\boldsymbol{\omega}(k)$ is a white Gaussian process noise. But as told before, in this paper we are considering maneuvering targets, so (9) is not suitable for our problem and we have to introduce another dynamic state equation for our purpose. 
What is proposed and introduced in this section is AIE based on the IE approach and is a new approach that operates in both the non-maneuver and maneuver modes and does not need any maneuver detection procedure. In this model, acceleration is treated as an additive input term in the system equation. So the system model of the target with maneuver is written as

$$
\begin{gathered}
\boldsymbol{X}(k+1)=\boldsymbol{A}(k) \boldsymbol{X}(k)+\boldsymbol{C}(k) \boldsymbol{u}(k)+\boldsymbol{B}(k) \boldsymbol{\omega}(k) \\
\boldsymbol{z}(k)=\boldsymbol{H}(k) \boldsymbol{X}(k)+\boldsymbol{v}(k)
\end{gathered}
$$

and the acceleration input vector is

$$
\boldsymbol{u}(k)=\left[\begin{array}{ll}
a_{x}(k) & a_{y}(k)
\end{array}\right]^{\mathrm{T}} .
$$

It is assumed that the acceleration $\boldsymbol{u}$ is a commonly unknown input which models the target maneuvers, when there is no maneuver, $\boldsymbol{u}$ is zero. In (10) and (11), the time dependent matrices $\boldsymbol{A}, \boldsymbol{B}, \boldsymbol{C}$ and $\boldsymbol{H}$ are as follows:

$$
\begin{gathered}
\boldsymbol{A}=\left[\begin{array}{cccc}
1 & T & 0 & 0 \\
0 & 1 & 0 & 0 \\
0 & 0 & 1 & T \\
0 & 0 & 0 & 1
\end{array}\right] \\
\boldsymbol{B}=\boldsymbol{C}=\left[\begin{array}{cccc}
T^{2} / 2 & T & 0 & 0 \\
0 & 0 & T^{2} / 2 & T
\end{array}\right]^{\mathrm{T}} \\
\boldsymbol{H}=\left[\begin{array}{cccc}
1 & 0 & 0 & 0 \\
0 & 0 & 1 & 0
\end{array}\right]
\end{gathered}
$$

where $\boldsymbol{\omega}(k)$ and $\boldsymbol{\nu}(k)$ are white process and measurement noises with zero means and covariances $Q$ and $\boldsymbol{R}$ respectively. Implicitly, these two noises are assumed to be uncorrelated so that for both $k_{1}$ and $k_{2}$ we have $\mathrm{E}\left[\boldsymbol{\omega}\left(k_{1}\right) \boldsymbol{v}^{\mathrm{T}}\left(k_{2}\right)\right]=0$. Also $\boldsymbol{\omega}(k)$ is a stochastic plant noise and $\boldsymbol{u}(k)$ is a deterministic but unknown additive maneuver term.

Now, if the additive maneuver $\boldsymbol{u}(k)$ is introduced as a new state, then the maneuvering model (10) and (11) can be converted to a non-maneuvering model with an augmented state equation in the form of the standard Bayesian mode (9) as follows:

$$
\begin{gathered}
{\left[\begin{array}{c}
\boldsymbol{X}(k+1) \\
\boldsymbol{u}(k+1)
\end{array}\right]=\left[\begin{array}{cc}
\boldsymbol{A} & \boldsymbol{C} \\
\mathbf{0} & \boldsymbol{I}
\end{array}\right]\left[\begin{array}{c}
\boldsymbol{X}(k) \\
\boldsymbol{u}(k)
\end{array}\right]+\left[\begin{array}{c}
\boldsymbol{B} \\
\boldsymbol{I}
\end{array}\right] \boldsymbol{\omega}(k)} \\
\boldsymbol{z}(k)=\boldsymbol{H}(k) \boldsymbol{X}(k)+\boldsymbol{v}(k) .
\end{gathered}
$$

New augmented vector and matrices are defined as

$$
\begin{gathered}
\boldsymbol{X}_{\text {aug }}(k)=\left[\begin{array}{c}
\boldsymbol{X}(k) \\
\boldsymbol{u}(k)
\end{array}\right], \quad \boldsymbol{A}_{\text {aug }}=\left[\begin{array}{cc}
\boldsymbol{A} & \boldsymbol{C} \\
\mathbf{0} & \boldsymbol{I}
\end{array}\right], \\
\boldsymbol{B}_{\text {aug }}=\left[\begin{array}{c}
\boldsymbol{B} \\
\boldsymbol{I}
\end{array}\right], \quad \boldsymbol{W}_{\text {aug }}=\boldsymbol{\omega} .
\end{gathered}
$$

Also the innovative posteriori measurement $\boldsymbol{z}(k+1)$ can be written as

$$
\begin{gathered}
\boldsymbol{z}(k+1)=\boldsymbol{H} \boldsymbol{X}(k+1)+\boldsymbol{v}(k+1)= \\
\boldsymbol{H}(\boldsymbol{A} \boldsymbol{X}(k)+\boldsymbol{C u}(k)+\boldsymbol{B} \boldsymbol{\omega}(k))+\boldsymbol{v}(k+1) \\
\boldsymbol{z}(k+1)=\left[\begin{array}{ll}
\boldsymbol{H} \boldsymbol{A} & \boldsymbol{H} \boldsymbol{C}
\end{array}\right]\left[\begin{array}{c}
\boldsymbol{X}(k) \\
\boldsymbol{u}(k)
\end{array}\right]+\boldsymbol{H} \boldsymbol{B} \boldsymbol{\omega}(k)+\boldsymbol{v}(k+1) .
\end{gathered}
$$

Thus, $\boldsymbol{H}_{\text {aug }}, \quad \boldsymbol{V}_{\text {aug }}$ and finally standard nonmaneuvering augmented state model are derived as

$$
\begin{gathered}
\boldsymbol{H}_{\text {aug }}=\left[\begin{array}{ll}
\boldsymbol{H} \boldsymbol{A} & \boldsymbol{H} \boldsymbol{C}
\end{array}\right] \\
\boldsymbol{V}_{\text {aug }}(k)=\boldsymbol{H} \boldsymbol{B} \boldsymbol{\omega}(k)+\boldsymbol{v}(k+1) \\
\boldsymbol{X}_{\text {aug }}(k+1)=\boldsymbol{A}_{\text {aug }} \boldsymbol{X}(k)+\boldsymbol{B}_{\text {aug }} \boldsymbol{W}_{\text {aug }}(k) \\
\boldsymbol{Z}_{\text {aug }}(k)=\boldsymbol{z}(k+1)=\boldsymbol{H}_{\text {aug }}(k) \boldsymbol{X}_{\text {aug }}(k)+\boldsymbol{V}_{\text {aug }}(k)
\end{gathered}
$$

where $\boldsymbol{X}_{\text {aug }}, \boldsymbol{A}_{\text {aug }}, \boldsymbol{B}_{\text {aug }}, \boldsymbol{W}_{\text {aug }}$ and $\boldsymbol{H}_{\text {aug }}, \boldsymbol{V}_{\text {aug }}, \boldsymbol{Z}_{\text {aug }}$ are obtained as (15), (17) and (18).

We can see that the augmented measurement noise $\boldsymbol{V}_{\text {aug }}(k)=\boldsymbol{H} \boldsymbol{B} \boldsymbol{\omega}(k)+\boldsymbol{v}(k+1)$ and the process noise $\boldsymbol{\omega}$ have time correlation with each other. Hence, in augmented measurement (19) and (20), a cross term is created between measurement and process noises. $\boldsymbol{T}_{\text {aug }}$ is called the cross covariance between augmented measurement noise $\boldsymbol{V}_{\text {aug }}$ and augmented process noise $\boldsymbol{W}_{\text {aug }}$ and calculated as follows:

$$
\begin{aligned}
\boldsymbol{T}_{\text {aug }}(k)=\mathrm{E}\left\{\boldsymbol{W}_{\text {aug }}(k) \boldsymbol{V}_{\text {aug }}^{\mathrm{T}}(k)\right\} & = \\
\mathrm{E}\left\{\boldsymbol{\omega}(k)[\boldsymbol{H} \boldsymbol{B} \boldsymbol{\omega}(k)+\boldsymbol{v}(k+1)]^{\mathrm{T}}\right\} & = \\
\mathrm{E}\left\{\boldsymbol{\omega}(k) \boldsymbol{\omega}^{\mathrm{T}}(k) \boldsymbol{B}^{\mathrm{T}} \boldsymbol{H}^{\mathrm{T}}\right\}+\mathrm{E}\left\{\boldsymbol{\omega}(k) \boldsymbol{v}(k+1)^{\mathrm{T}}\right\} & =\boldsymbol{Q} \boldsymbol{B}^{\mathrm{T}} \boldsymbol{H}^{\mathrm{T}} .
\end{aligned}
$$

We remember that $\boldsymbol{v}(k)$ and $\boldsymbol{\omega}(k)$ are uncorrelated white processes and $\boldsymbol{V}_{\text {aug }}$ is still a white process. Thus, $\boldsymbol{Q}_{\text {aug }}(k)$ and $\boldsymbol{R}_{\text {aug }}(k)$ are computed as follows:

$$
\begin{gathered}
\boldsymbol{Q}_{\text {aug }}(k)=\mathrm{E}\left\{\boldsymbol{W}_{\text {aug }}(k) \boldsymbol{W}_{\text {aug }}^{\mathrm{T}}(k)\right\}=\mathrm{E}\left\{\boldsymbol{\omega}(k) \boldsymbol{\omega}^{\mathrm{T}}(k)\right\}=\boldsymbol{Q} \\
\boldsymbol{R}_{\text {aug }}(k)=\mathrm{E}\left\{\boldsymbol{V}_{\text {aug }}(k) \boldsymbol{V}_{\text {aug }}^{\mathrm{T}}(k)\right\}= \\
\left.\mathrm{E}\{(\boldsymbol{H} \boldsymbol{B} \boldsymbol{\omega}(k)+\boldsymbol{v}(k+1)) \boldsymbol{H} \boldsymbol{B} \boldsymbol{\omega}(k)+\boldsymbol{v}(k+1))^{\mathrm{T}}\right\}= \\
\boldsymbol{H} \boldsymbol{B E}\left\{\boldsymbol{\omega}(k) \boldsymbol{\omega}(k)^{\mathrm{T}}\right\} \boldsymbol{B}^{\mathrm{T}} \boldsymbol{H}^{\mathrm{T}}+\mathrm{E}\left\{\boldsymbol{v}(k+1) \boldsymbol{v}(k+1)^{\mathrm{T}}\right\} \\
\boldsymbol{R}_{\text {aug }}(k)=\mathrm{E}\left\{\boldsymbol{V}_{\text {aug }}(k) \boldsymbol{V}_{\text {aug }}^{\mathrm{T}}(k)\right\}=\boldsymbol{H} \boldsymbol{B} \boldsymbol{Q} \boldsymbol{B}^{\mathrm{T}} \boldsymbol{H}^{\mathrm{T}}+\boldsymbol{R}
\end{gathered}
$$

$\mathrm{E}\left(\boldsymbol{W}_{\text {aug }}\left(k_{1}\right) \boldsymbol{V}_{\text {aug }}^{\mathrm{T}}\left(k_{2}\right)\right)=\left\{\begin{array}{l}{\left[\begin{array}{cc}\boldsymbol{Q}_{\text {aug }} & \boldsymbol{T}_{\text {aug }} \\ \boldsymbol{T}_{\text {aug }}^{\mathrm{T}} & \boldsymbol{R}_{\text {aug }}\end{array}\right], \quad k_{1}=k_{2}} \\ 0, \quad k_{1} \neq k_{2}\end{array}\right.$.

In the following, the structure of the Kalman filter for the AIE is presented and we see that the most important 
difference between its equations and simple Kalman filter equations is the effect of $\boldsymbol{T}_{\text {aug }}$ and also it is seen that the new Kalman gain is modified on the basis of $\boldsymbol{T}_{\text {aug }}$ and the optimal target maneuver estimator for the augmented system (14) and according to the new Kalman gain is

$$
\begin{gathered}
\widehat{\boldsymbol{X}}_{\mathrm{aug}}(k+1 \mid k+1)= \\
\widehat{\boldsymbol{X}}_{\mathrm{aug}}(k+1 \mid k)+\boldsymbol{K}_{\mathrm{aug}}(k+1) \boldsymbol{r}_{\mathrm{aug}}(k+1) .
\end{gathered}
$$

\section{Prediction:}

$$
\begin{gathered}
\widehat{\boldsymbol{X}}_{\text {aug }}(k+1 \mid k)=\boldsymbol{A}_{\text {aug }} \widehat{\boldsymbol{X}}_{\text {aug }}(k \mid k) \\
\boldsymbol{P}_{\text {aug }}(k+1 \mid k)=\boldsymbol{A}_{\text {aug }} \boldsymbol{P}_{\text {aug }}(k \mid k) \boldsymbol{A}_{\text {aug }}^{\mathrm{T}}+\boldsymbol{B}_{\text {aug }} \boldsymbol{Q}_{\text {aug }} \boldsymbol{B}_{\text {aug }}^{\mathrm{T}}
\end{gathered}
$$

Estimation:

$$
\begin{gathered}
\boldsymbol{r}_{\text {aug }}(k+1)=\boldsymbol{z}_{k+1}-\boldsymbol{H}_{\text {aug }} \widehat{\boldsymbol{X}}(k+1 \mid k) \\
\boldsymbol{S}_{\text {aug }}(k+1)=\boldsymbol{R}_{\text {aug }}+\boldsymbol{H}_{\text {aug }} \boldsymbol{P}_{\text {aug }}(k+1 \mid k) \boldsymbol{H}_{\text {aug }}^{\mathrm{T}} \\
\boldsymbol{K}_{\text {aug }}(k+1)=\left[\boldsymbol{P}_{\text {aug }}(k+1 \mid k) \boldsymbol{H}_{\text {aug }}^{\mathrm{T}}+\boldsymbol{B}_{\text {aug }} \boldsymbol{T}_{\text {aug }}\right] \boldsymbol{S}_{\text {aug }}^{-1}(k+1) \\
\boldsymbol{P}_{\text {aug }}(k+1 \mid k+1)=\boldsymbol{P}_{\text {aug }}(k+1 \mid k)-\boldsymbol{P}_{\text {aug }}(k+1 \mid k) . \\
\boldsymbol{H}_{\text {aug }}^{\mathrm{T}}(k+1)\left[\boldsymbol{R}_{\text {aug }}+\boldsymbol{H}_{\text {aug }} \boldsymbol{P}_{\text {aug }}(k+1 \mid k)_{\text {aug }} \boldsymbol{H}_{\text {aug }}^{\mathrm{T}}\right]^{-1} . \\
\boldsymbol{H}_{\text {aug }} \boldsymbol{P}_{\text {aug }}(k+1 \mid k)
\end{gathered}
$$

Fig. 4 illustrates the final algorithm for AIE in the MTT problem. As can be seen in this figure, MTT is based on two steps, the first one is data association (MCMCDA) and the second one is estimation and tracking.

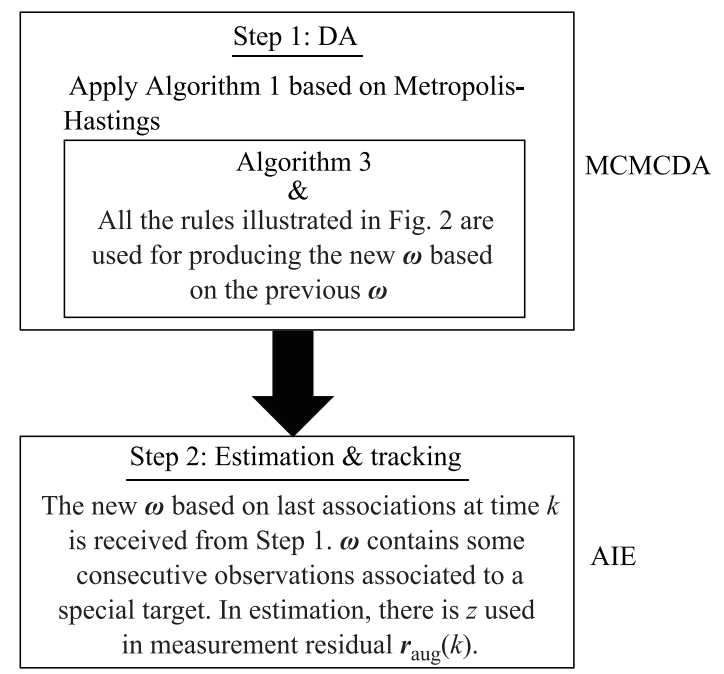

Fig. 4 AIE in MTT

In Step 1, the Metropolis-Hastings method and all rules described before are exerted to receive most probable associations for targets and observations. After that in Step 2, these observations are used in AIE Kalman filter to derive the best estimations in two cases of maneuvering and nonmaneuvering targets motions.

\section{Simulations and results}

In this section, we want to compare our proposed model AIE-MCMCDA with other models of MTT. As we know, the performances of MTT algorithms can be compared to each other in two approaches of known number and unknown number of targets. Among old models of multi target tracking, JPDA is the well-known and most famous in the approach of known number of targets. In [7], it is shown that MCMCDA can have the same performance to JPDA with lower numbers of samples and lower processing time. So in this approach, we compare only different types of estimation with the same data association model of MCMCDA.

Case 1 The number of targets is known

In this case, four different types of estimation, AIE, IMM, constant acceleration (CA) and constant velocity (CV) (in the presence of the same data association model of MCMCDA), are compared to each other for the root mean square (RMS) errors in tracking parameters. We consider that the targets are moving in a surveillance region $R=[0,1000] \times[0,1000]$. The state vector for the $\mathrm{CV}$ model is $[x, y, \dot{x}, \dot{y}]^{\mathrm{T}}$ and for CA and two maneuver models of IMM is $[x, y, \dot{x}, \dot{y}, \ddot{x}, \ddot{y}]^{\mathrm{T}}$. Our IMM model is a combination of one non-maneuver model with two maneuvering models and finally the state vector for AIE is $\left[x, y, \dot{x}, \dot{y}, u_{x}, u_{y}\right]^{\mathrm{T}}$.

The state and measurement equations for $\mathrm{CV}, \mathrm{CA}$ and IMM are as follows:

$$
\left\{\begin{array}{l}
\boldsymbol{X}(k+1)=\boldsymbol{A} \boldsymbol{X}(k)+\boldsymbol{B} \boldsymbol{\omega}(k) \\
\boldsymbol{z}(k)=\boldsymbol{H} \boldsymbol{X}(k)+\boldsymbol{v}(k)
\end{array}\right.
$$

and for AIE the equations are as follows:

$$
\left\{\begin{array}{l}
\boldsymbol{X}_{\text {aug }}(k+1)=\boldsymbol{A}_{\text {aug }} \boldsymbol{X}_{\text {aug }}(k)+\boldsymbol{B}_{\text {aug }} \boldsymbol{\omega}_{\text {aug }}(k) \\
\boldsymbol{z}_{\text {aug }}(k)=\boldsymbol{H}_{\text {aug }} \boldsymbol{X}_{\text {aug }}(k)+\boldsymbol{v}_{\text {aug }}(k)
\end{array}\right.
$$

where $(x, y)$ is the target position along the $x$ and $y$ axes and also $(\dot{x}, \dot{y})$ is the velocity vector. $(\ddot{x}, \ddot{y})$ and/or $\left(u_{x}, u_{y}\right)$ are accelerations along the usual $x$ and $y$ axes.

$T=1 \mathrm{~s}$ is the sampling period, $\boldsymbol{\omega}(k)$ and $\boldsymbol{v}(k)$ are zeromean Gaussian process and measurement noises with the covariances $\boldsymbol{Q}=\operatorname{diag}(100,100)$ and $\boldsymbol{R}=\operatorname{diag}(100,100)$ respectively. In this example, six targets are moving in the region $R$ with maneuver and suppose that $p_{d}=0.9$, $\lambda_{f}=0.0001$, the targets are tracked for 100 scans and the scenario is repeated 100 times.

The first scan of each track is set on the correct reports. In Table 1, the performances of these four estimation models are compared. Comparisons are based on the RMSE 
averages in four different parts of surveillance time [0$25 \mathrm{~s}],[25-50 \mathrm{~s}],[50-75 \mathrm{~s}]$ and $[75-100 \mathrm{~s}]$. As seen in this table, approximately in all cases, the average mean square error (RMSE) for AIE is better than all other three models except in few cases like duration time [50-75 $\mathrm{s}$ ] that in targets 2 and 5, the IMM estimation model has better performance than AIE.

Table 1 RMS position error for different estimation methods with MCMCDA

\begin{tabular}{|c|c|c|c|c|}
\hline Method & $0-25 \mathrm{~s}$ & $25-50 \mathrm{~s}$ & $50-75 \mathrm{~s}$ & $75-100 \mathrm{~s}$ \\
\hline AIE & 3.5984 & 1.0714 & 1.2624 & 1.2186 \\
\hline IMM & 4.7575 & 1.5094 & 2.4174 & 1.2473 \\
\hline CA & 4.517 & 2.3208 & 1.8051 & 1.5125 \\
\hline $\mathrm{CV}$ & 11.726 & 7.3552 & 6.5921 & 16.34 \\
\hline \multicolumn{5}{|c|}{ Target 2: Average RMS position errors } \\
\hline Method & $0-25 \mathrm{~s}$ & $25-50 \mathrm{~s}$ & $50-75 \mathrm{~s}$ & $75-100 s$ \\
\hline AIE & 4.5287 & 1.4339 & 0.8596 & 0.7315 \\
\hline IMM & 6.012 & 2.8757 & 0.8036 & 2.1631 \\
\hline CA & 5.6961 & 3.3631 & 1.4523 & 1.0737 \\
\hline $\mathrm{CV}$ & 14.054 & 9.3525 & 9.4017 & 13.278 \\
\hline \multicolumn{5}{|c|}{ Target 3: Average RMS position errors } \\
\hline Method & $0-25 \mathrm{~s}$ & $25-50 \mathrm{~s}$ & $50-75 \mathrm{~s}$ & $75-100 \mathrm{~s}$ \\
\hline AIE & 3.4156 & 1.1002 & 0.6928 & 0.9793 \\
\hline IMM & 4.1593 & 1.4826 & 0.7140 & 1.36 .9 \\
\hline $\mathrm{CA}$ & 4.2533 & 1.7012 & 0.6195 & 1.0741 \\
\hline $\mathrm{CV}$ & 10.355 & 5.6075 & 6.816 & 10.04 \\
\hline \multicolumn{5}{|c|}{ Target 4: Average RMS position errors } \\
\hline Method & $0-25 \mathrm{~s}$ & $25-50 \mathrm{~s}$ & $50-75 \mathrm{~s}$ & $75-100 \mathrm{~s}$ \\
\hline AIE & 4.0461 & 1.8623 & 1.0872 & 1.0824 \\
\hline IMM & 5.0201 & 3.2237 & 1.5007 & 1.2834 \\
\hline $\mathrm{CA}$ & 4.9397 & 3.5467 & 2.0148 & 1.2314 \\
\hline $\mathrm{CV}$ & 11.655 & 10.198 & 7.5291 & 4.8073 \\
\hline \multicolumn{5}{|c|}{ Target 5: Average RMS position errors } \\
\hline Method & $0-25 \mathrm{~s}$ & $25-50 \mathrm{~s}$ & $50-75 \mathrm{~s}$ & $75-100 \mathrm{~s}$ \\
\hline AIE & 4.1705 & 1.7831 & 0.8591 & 0.7489 \\
\hline IMM & 5.7737 & 3.3062 & 0.8261 & 0.8627 \\
\hline $\mathrm{CA}$ & 5.418 & 3.5588 & 1.3613 & 0.9265 \\
\hline $\mathrm{CV}$ & 15.298 & 9.6313 & 14.045 & 4.7554 \\
\hline \multicolumn{5}{|c|}{ Target 6: Average RMS position errors } \\
\hline Method & $0-25 \mathrm{~s}$ & $25-50 \mathrm{~s}$ & $50-75 \mathrm{~s}$ & $75-100 \mathrm{~s}$ \\
\hline AIE & 4.1283 & 1.4399 & 1.0685 & 0.3988 \\
\hline IMM & 5.2328 & 2.9672 & 1.468 & 1.1833 \\
\hline CA & 5.084 & 3.1482 & 1.7744 & 0.7214 \\
\hline $\mathrm{CV}$ & 13.132 & 6.2792 & 9.3407 & 4.2072 \\
\hline
\end{tabular}

Case 2 The number of targets is unknown

In this case, the performances of AIE-MCMCDA, MHT [21] and nearest neighbor filter (NNF) [22] are compared with each other. Surveillance region, state vectors and state and measurement equations are defined as Case 1. Three major parameters are so effective in the complexity of MTT problems.

These parameters are false alarm rate $\lambda_{f}, p_{d}$ and tracks density. Increasing $\lambda_{f}$ and tracks density and/or decreasing $p_{d}$ can make the problem more complicated.

By varying these mentioned parameters, we can compare the performances of AIE-MCMCDA, NNF and MHT.
For easier comparison, the MAP approach is used in which the targets states are estimated from $\widehat{\omega}$ computed from the MCMCDA algorithm.

As we know, the number of targets $J$ is not fixed, so to use a standard model such as the mean square error is not practical to measure the performance of an algorithm. Hence two different metrics are used to measure performance: $\epsilon_{J}$ as the estimation error in number of targets and $\epsilon_{X}$ as the estimation error in position. Suppose that $J^{*}(k)$ is the number of targets at the time $k T . \in_{J}$ is defined as follows [22]:

$$
\epsilon_{J}=\frac{1}{\sum J^{*}(k)} \sum_{k=1}^{K}\left|J(k)-J^{*}(k)\right| .
$$

The computation of $\epsilon_{X}$ is done when it makes sense and the maximum number of common tracks in any $k$ is $M(k)=\min \left(J(k), J^{*}(k)\right)$. Based on positions at $k-1$, $k, k+1, M(k)$ number of matches can be found between true tracks and estimated tracks. But for each match $i$, suppose $x_{i}^{*}(k)$ be the position of true track and $x_{i}(k)$ be the estimated track at the time $k . \in_{X}$ can be defined as follows [22]:

$$
\epsilon_{X}^{2}=\frac{1}{\sum M(k)} \sum_{k=1}^{K} \sum_{i=1}^{M(k)}\left\|x_{i}(k)-x_{i}^{*}(k)\right\|^{2} .
$$

For easier comparison, we normalize $\epsilon_{J}$ and $\epsilon_{X}$ based on the number of targets and do the simulations on a PC with a $3 \mathrm{GHz}$ Intel processor.

(i) Number of tracks. In this case, we vary $J$ (the number of tracks) from 10 to 100 . The other parameters are held fixed: $R=[0,1000] \times[0,1000], T_{\text {fin }}=100 \mathrm{~s}$, $P_{d}=0.9, V=1, V=J / T_{\text {fin }}, p_{z}=0.0001, \bar{d}=5 \mathrm{~m}$, $\bar{v}=100 \mathrm{~m} / \mathrm{s}, \bar{a}=0.5 \mathrm{~m} / \mathrm{s}^{2}$. In any $k, 10$ test cases are generated randomly. NNF can be used to computation of AIEMCMCDA initial states and AIE-MCMCDA uses 50000 samples. For any $k$, we compute $\epsilon_{J}$ and $\epsilon_{X}$ from the 10 test cases. Additionally, we also average over 10 runs per test case for AIE-MCMCDA and also shown in Fig. 5(a) and Fig. 5(b) respectively.

As we expected and seen in Fig. 5(a), in estimation error for number of targets, AIE-MCMCDA has better performance against other algorithms and MHT is approximately like AIE-MCMCDA. Likewise, MHT has superior performance than NNF in this case.

But Fig. 5(b) shows us that in position estimation error, because of pruning, if the number of tracks increases, the performance of MHT decreases and AIE-MCMCDA shows superior performance against both MHT and NNF. In this example, since all targets are presented from $t=$ $T_{\text {init }}$ to $t=T_{\text {fin }}$, then NNF shows better performances than MHT at higher numbers of targets. 


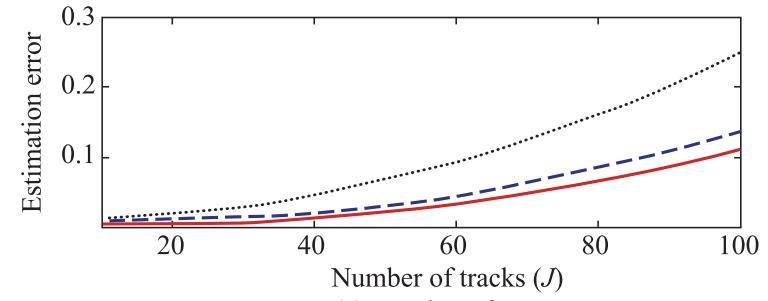

(a) Number of targets

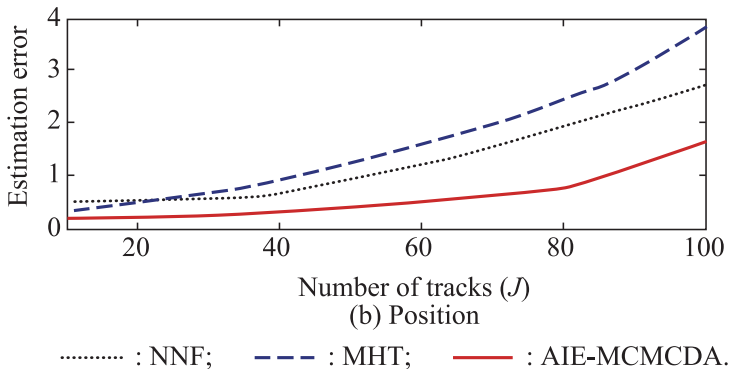

Fig. 5 Estimation error in number of targets and position vs. number of tracks for NNF, MHT and AIE-MCMCDA

(ii) False alarms. In this case, by considering the settings same as the previous case, only the false alarm rate changes from $V=1$ to $V=100$ with an increment of 10. Notice that the number of tracks is fixed at $J=10$. For each value of $V, 50000$ samples are used and randomly generate 10 test cases and AIE-MCMCDA is run 10 times per test case. The estimation errors for the number of targets and for the position and also for three algorithms at different false alarm rates are given in Fig. 6(a) and Fig. 6(b). The results show that AIE-MCMCDA performs well at high false alarm rates in both cases of number of targets estimation error and position estimation error.

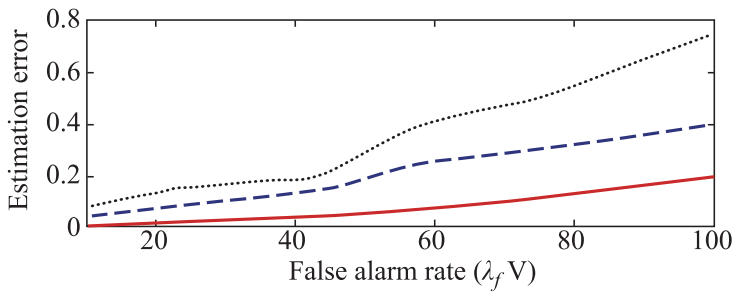

(a) Number of targets

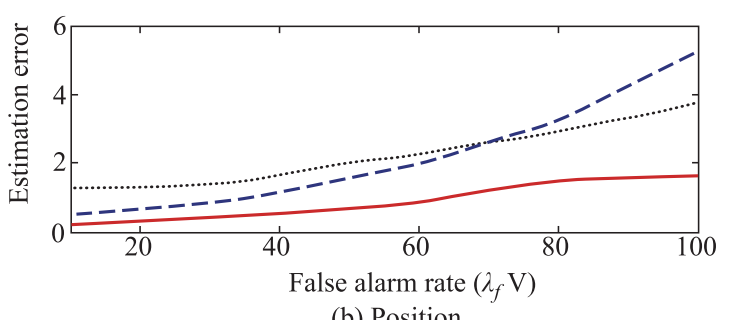

(b) Position

$$
\text { ......... : NNF; - - - : MHT; — : AIE-MCMCDA. }
$$

Fig. 6 Estimation error in number of targets and position vs. false alarm rate for NNF, MHT and AIE-MCMCDA

But in position estimation error, MHT does not have acceptable performance for higher values of false alarms and becomes hopelessly confused. (iii) Detection probability. In this case, we vary the detection probability from 0.1 to 0.99 with 0.1 step and suppose that the number of tracks is $J=10$ and the false alarm rate is $V=1$. Also 50000 samples are used for AIE-MCMCDA and 10 test cases are randomly generated. In Fig. 7(a) and Fig. 7(b) and for three different algorithms, the errors $\in_{J}$ and $\in_{X}$ at different detection probabilities are illustrated. In estimation error $\in_{J}$, the overall performance of AIE-MCMCDA is better than that of MHT and NNF but in middle values of $p_{d}$, NNF is better than MHT and in estimation error $\in_{X}$, MHT performs lightly better than MCMCDA only at high values of detection probability $\left(p_{d}>0.9\right)$ and finally the NNF algorithm performs very poorly.

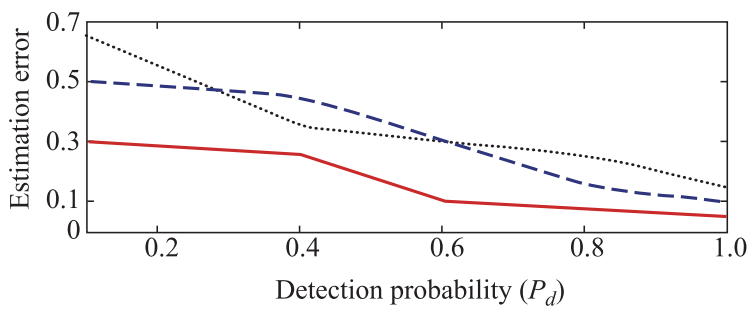

(a) Number of targets

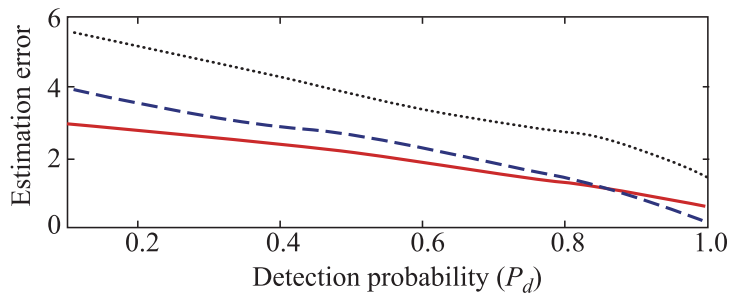

(b) Position

Fig. 7 Estimation error in number of targets and position vs. detection probability for NNF, MHT and AIE-MCMCDA

Although in Cases 2 and 3, the number of targets is fixed $\left(J^{*}=10\right)$, but variation of the false alarm and the detection probability have serious effects on estimated number of targets $J$. So it is reasonable that $\in_{J}$ and $\in_{X}$ have significant changes with different values of $V$ and $p_{d}$. AIEMCMCDA and MHT can be compared with their running times (Fig. 8).

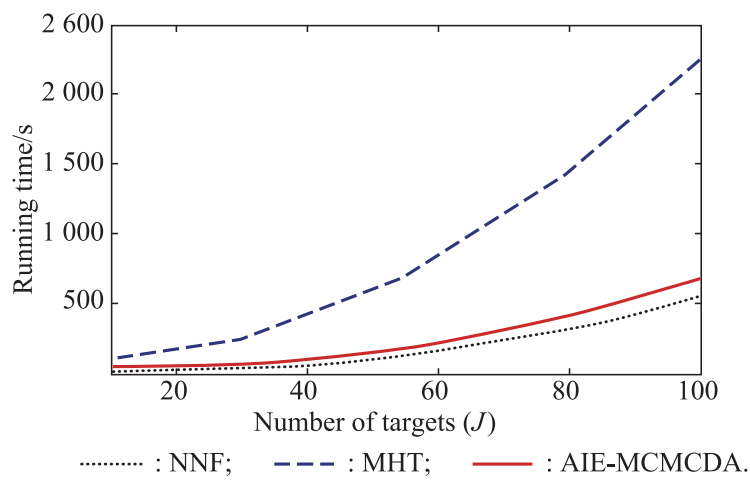

Fig. 8 Running times for different MTT methods 
In AIE-MCMCDA, the number of observations and the number of samples can regulate the running time but in MHT, depends only on the problem complexity which is not predictable.

Additionally, the required memory for AIE-MCMCDA is significantly less than what required in MHT because in AIE-MCMCDA one association is stored in any time.

\section{Conclusions}

In this paper, we have presented AIE-MCMCDA for solving multiple maneuvering targets tracking in a cluttered environment. As mentioned before, MTT in general form has two main bases: DA and estimation. In DA, MCMCDA has been introduced as a superior algorithm than older algorithms. What happens in MCMCDA is to sample randomly around the region with more concentrated posterior instead of considering all the associations.

MCMCDA has shown that it has better performance in simple case of the known number of targets and more general form of the unknown number of targets that appear and disappear in random times. MCMCDA is capable of initiating and terminating tracks and can have better performances with lower process time and more rapid convergence. In estimation part of the problem, a new model of IE called AIE is proposed for maneuvering target tracking that changes the dynamic equation to a standard Kalman filter. AIE is based on the mixed Bayesian-Fisher uncertainty model and converts the maneuvering dynamic equation to a non-maneuvering dynamic case. This converting is based on a special state augmentation for deriving a standard Bayesian model. In this algorithm, we do not need any maneuver detector and therefore no time consumed for maneuver detection. Simulation results show that even in difficult conditions like low detection probabilities, and high false alarm rates or large number of targets in a dense environment, AIE-MCMCDA has better performance against MHT and NNF algorithms.

Both AIE and MCMCDA algorithms are based on infinite impulse response (IIR) filtering. Although according to the results of this paper, AIE and MCMCDA have better performances compared to other models, but it should be noticed that the IIR structure cannot be robust against temporary model uncertainties like deviation in sampling time or noise variances. So for future work we should use robust estimation and DA models instead of IIR models. Also in future work, colored and multiplied noises can be considered instead of white Gaussian noises.

\section{References}

[1] MCINTYRE G A, HINTZ K J. Comparison of several maneuver tracking models. Proc. of the SPIE Conference Signal Processing, Sensory Fusion and Target Recognition VII, 1998:
$48-63$.

[2] KHALOOZADEH H, KARSAZ A. Modified input estimation technique for tracking manoeuvring targets. IET Radar, Sonar \& Navigation, 2008, 3(1): 30-41.

[3] CHAN Y T, HU A G C, PLANT J B. A Kalman filter based tracking scheme with input estimation. IEEE Trans. on Aerospace and Electronic Systems, 2007, 15(2): 237-244.

[4] BOGLER P L. Tracking a maneuvering target using input estimation. IEEE Trans. on Aerospace \& Electronic Systems, 1987, 23(3): 298-310.

[5] WHANG I H, LEE J G, SUNG T K. Modified input estimation technique using pseudoresiduals. IEEE Trans. on Aerospace and Electronic Systems, 2002, 30(1): 220-228.

[6] LEE H, TAHK M J. Generalized input-estimation technique for tracking maneuvering targets. IEEE Trans. on Aerospace and Electronic Systems, 1999, 35(4): 1388-1402.

[7] OH S, RUSSELL S, SASTRY S. Markov chain Monte Carlo data association for multi-target tracking. IEEE Trans. on Automatic Control, 2009, 54(3): 481 - 497.

[8] REID D. An algorithm for tracking multiple targets. IEEE Trans. on Automatic Control, 1979, 24(6): 843-854.

[9] BAR-SHALOM Y, FORTMANN T. Tracking and data association. San Diego, CA: Academic Press, 1988.

[10] PASUlA H, RUSSELl S J, OSTLAND M, et al. Tracking many objects with many sensors. Proc. of the 16th International Joint Conference on Artificial Intelligence, 1999: 11601171.

[11] PASULA H. Identity uncertainty. Berkeley CA: University of California, 2003.

[12] CONG S, HONG L, WICKER D. Markov-chain MonteCarlo approach for association probability evaluation. IEE Proceedings-Control Theory and Applications, 2004, 151(2): $185-193$.

[13] DELLAERT F, SEITZ S M, THORPE C E, et al. EM, MCMC, and chain flipping for structure from motion with unknown correspondence. Machine Learning, 2003, 50(1-2): 45-71.

[14] BERGMAN N, DOUCET A. Markov chain Monte Carlo data association for target tracking. Proc. of the IEEE International Conference on Acoustics Speech, Signal Processing, 2000: $11705-11708$.

[15] BAR-SHALOM Y. Multi-target multi-sensor tracking: advanced applications. Norwood, Mass: Artech House, 1990.

[16] HASTINGS W. Monte Carlo sampling methods using Markov chains and their applications. Biometrika, 1970, 57(1): $97-$ 109.

[17] OH S. A scalable multi-target tracking algorithm for wireless sensor networks. International Journal of Distributed Sensor Networks, 2012, DOI:10.1155/2012/938521.

[18] GILKS W, RICHARDSON S, SPIEGELHALTER D. Interdisciplinary statistics. New York: Chapman and Hall, 1996.

[19] KHALOOZADEH H, KARSAZ A. A new state augmentation for maneuvering targets detection. Proc. of the Signal Processing and Communications, 2004: 65-69.

[20] KARSAZ A, KHALOOZADEH H. A new algorithm based on generalized target maneuver detection. Proc. of the 6th International Conference on Control and Automation, 2007, DOI:10.1109/ICCA.2007.4376918.

[21] COX I. Multiple hypothesis tracking code. http://www.ee.ucl. ac.uk/ icox/.

[22] DOUCET A, DE FREITAS J, GORDON N. Sequential Monte Carlo methods in practice. New York: Springer, 2001. 


\section{Biographies}

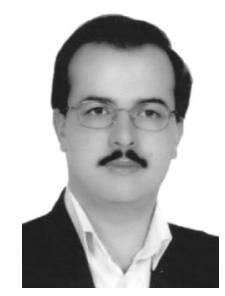

HADAEGH Mahmoudreza was born in 1975. He received his B.Sc. degree in electronics engineering from Shiraz University, Shiraz, Iran, in 1999, his M.Sc. degree in control engineering from K. N. Toosi University of Technology, Tehran, in 2001. $\mathrm{He}$ is currently a Ph.D. student in control engineering with the Department of Electrical and Computer Engineering, Tehran Science and Research Branch, Islamic Azad University, Tehran, Iran. Now he is an instructor in Islamic Azad University and his interest areas are in target tracking for single and multiple targets and wireless sensor networks.

E-mail: mr_hadaegh@yahoo.com

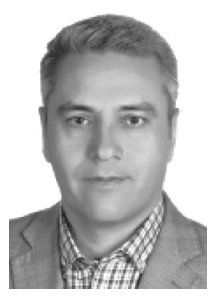

KHALOOZADEH Hamid was born in 1965. He received his B.Sc. degree in control engineering from Sharif University of Technology, Tehran, Iran, in 1990, his M.Sc. degree in control engineering from K. N. Toosi University of Technology, Tehran, in 1993, and his Ph.D. degree in control engineering from Tarbiat Modarres University, Tehran, in 1998. He is currently a professor with the Department of
Systems and Control, Faculty of Electrical Engineering at the K. N. Toosi University of Technology. He is the director of the Industrial Control Center of Excellence (ICCE), at K. N. Toosi University of Technology. His interest areas are stochastic estimation and control, system identification, optimal control, and time series analysis.

E-mail: h_khaloozadeh@kntu.ac.ir

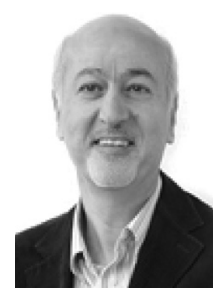

BEHESHTI Mohammadtaghi was born in 1958. He received his B.S. degree in electrical engineering from University of Nebraska at Lincoln in 1984, his M.S. and Ph.D. degrees in control engineering from Wichita State University, Wichita Kansas, in 1987 and 1992, respectively. He is an associate professor of Department of Electrical Engineering, Control Group, Tarbiat Modares University, Tehran, Iran since 1995. His research interests are in modeling and control of singular perturbation systems, modeling and control of communication and computer networks, non-conventional sources and microgrid systems.

E-mail: mbehesht@modares.ac.ir 